\title{
Intervention Model
}

National Cancer Institute

\section{Source}

National Cancer Institute. Intervention Model. NCI Thesaurus. Code C98746.

The trial design developed to compare treatment groups. 